\title{
Review
}

\section{Occupational Exposure of Medical Staff of a Tianjin Grade 3 Hospital to Human Immunodeficiency Virus in 2013-2015}

\author{
Wenlong $\mathrm{He}^{1}$, Chao Wen ${ }^{2}$, Xiaoyu Wang ${ }^{2}$ \\ ${ }^{1}$ TianJin Medical Univercity School of Public Health, TianJin, China \\ ${ }^{2}$ Department of Otorhinolaryngology Head and Neck Surgery, Tianjin First Center Hospital, TianJin, China
}

\author{
Keywords \\ HIV; Blood-borne pathogen; Medical staff; \\ Occupational exposure; Needle-stick injuries \\ Correspondence \\ Wenlong $\mathrm{He}$, \\ E-mail: hwltme_edu@163.com \\ DOI: 10.1515/ii-2017-0107
}

\begin{abstract}
Purpose: This study aims to gain insights into occupational exposure of medical staff to human immunodeficiency virus (HIV) and to provide effective precautionary measures to protect them against risks arising from blood-borne pathogens.

Methodology: Data on 46 confirmed HIV-infected patients were analyzed statistically. Results: Medical staff were exposed to blood-borne pathogens in 45 cases, and most were female and probationary nurses. Risks of occupational exposure of medical staff to HIV increased continuously as more HIV-infected patients were admitted by hospitals each year. Conclusion: Medical staff should receive information about HIV blood-borne pathogen infection of patients, shorten the window period for HIV exposure, and practice specified precautionary measures and cut down risks of exposure to HIV.
\end{abstract}

Human immunodeficiency virus (HIV) can lead to acquired immune deficiency syndrome (AIDS). HIV is mainly spread through blood contact, sexual contact, transmission from mother to fetus, and other approaches [1]. To date, major medical and health organizations in all levels screen out HIV cases ${ }^{[2,3]}$. A significant number of patients may receive outpatient services and be hospitalized in Grade 3 hospitals. Thus, needle-stick injuries occur occasionally, and medical staff become highly exposed to HIV. HIV is the major blood-borne pathogen against which medical staff in China should be protected considering regulations in protection of medical staff of People's Republic of China against blood-borne pathogens during occupational contact ${ }^{[4]}$. For such reason, this study analyzes occupational exposure risks of medical staff of a Tianjin Grade 3 hospital to HIV in 2013-2015.

\section{Object and Methodology}

\section{Object}

In 2013 to 2015, 46 patients were diagnosed with HIV in a Grade 3 hospital in Tianjin based on medical examinations among patients suspected with HIV infection or inpatients who were suspected to suffer from HIV. At the same time, the
Infection Control Department recorded 45 medical workers, including physicians, nurses, probationers, those who were engaged in advanced studies, and logistics workers who were occupationally exposed to blood-borne pathogens. Medical staff were informed of patients' infection immediately after diagnosis with HIV.

\section{Methodology}

Statistical analyses were conducted on age, gender, preadmission HIV infection, duration of diagnosis of HIV infection, and other data of $46 \mathrm{HIV}$-infected patients from 2013 to 2015. Based on the Registration Form of Medical Staff Occupational Exposure to Blood-borne Pathogens completed by medical staff during risk exposure in 2013 -2015 this study provides a retrospective analysis of occupational exposure information of 45 medical workers.

\section{Data Processing and Statistical Analysis}

EXCEL was used for data processing, and constituent ratio was calculated.

\section{Results}

HIV confirmatory test 
A total of $46 \mathrm{HIV}$-infected patients were admitted by the hospital in 2013-2015, and two died of AIDS. Most patients were male, and male to female ratio was $6.7: 1$. Patients aged 19-63, with an average age of $36.25 \pm 10.21$ years old. Patients aged under 40 accounted for $65.2 \%$ (30/40), those aged between 41 and 60 accounted for $30.4 \%$, and those aged above 60 accounted for $4.3 \%(2 / 46)$ of total cases. Among these 46 patients, six received outpatient services, whereas 40 were hospitalized. One patient stated that he was infected with HIV when he received outpatient services. The remaining patients were unaware of their HIV infection or concealed their condition. Based on data regarding admission time of patients and time of validation of HIV-infected condition, the hospital took an average of 4.5 days to detect HIV-infected patients starting from admission time. Compared with the abovementioned time, an average of 2.6 days were required to preliminarily confirm HIV infection among outpatients. An average of 4.9 days were needed to preliminarily confirm HIV infection fact among inpatients.

\section{Confirmation of occupational exposure of medical staff to blood-borne pathogens}

A total of 45 medical workers who were exposed to bloodborne pathogens were recorded by the hospital during their work from 2013 to 2015, and their average age was 26.14 3.65 years old. Most medical workers were females, and male to female ratio was $1: 4$. Up to $80 \%$ of incidents occurred during nursing operation. Thus, high-risk population comprised nurses and probationary nurses. Occupational exposure of medical staff to blood-borne pathogens was mainly attributed to needle stick, accounting for $97.8 \%$ (44/45), and in one case, the medical worker was bitten by a patient. In 38 cases, medical workers were injured by hollow needles. In three cases, medical staff were injured by solid needles (one was injured by nail, one by suture, and one by a scalpel). Hands of medical staffs represented the major body parts exposed to risks, accounting for $84.4 \%$ (38/45) of exposure cases. In seven cases, patients' blood splashed into eyes of medical staff. A total of 22 cases were exposed to hepatitis B virus (HBV) (44.4\%), 14 cases were exposed to Treponema pallidum (31.1\%), six cases to HIV (13.33\%), three cases to hepatitis $\mathrm{C}$ virus (6.7\%), one case to both HBV and Treponema pallidum (2.2\%), and one case presented injury by sterilized medical apparatus (2.2\%).

\section{Occupational exposure of medical staff to HIV}

In 2013-2015, four HIV exposure incidents among medical staff occurred in the hospital, i.e., one case in 2013 and three cases in 2015. From follow-up visits, no medical workers were infected during incidents. For the number of HIV screening cases, Fig. 1 shows the number of preliminarily confirmed HIV-infected cases and occupational exposure of medical staff to HIV in three years. As more preliminarily confirmed HIV-infected patients were admitted by the hospital, occupational exposure risks of medical staff to HIV became significant.

\section{Discussion}

Needle stick is the main injury that hospital medical staff may experience while working, and nursing staff are the ones mainly exposed to such risks. This conclusion agrees with research findings of Li Wenyu and Xiang Peiying [5-8]. In 2013-2015, a sharp increase was observed in preliminarily confirmed HIV-infected patients admitted by the hospital, that is to say, from eight cases in 2013 to 26 cases in 2015. Increasing rate of preliminarily confirmed HIV-infected patients was apparently higher than increasing rate of patients screened during the same period. This result indicates that although AIDS still features a low epidemic trend, increasing number of living HIVinfected patients worsens conditions of HIV prevention ${ }^{[11}$ ${ }^{-13]}$. Probability of exposure of medical staff to HIV risks increased sharply. For outpatients, diagnosis period is relatively short when they are infected with HIV because of their usually definite goals when receiving outpatient services. However, in the case of inpatients, confirmation of HIV infection requires an average of 4.9 days because patients are hospitalized not because of AIDS, or they deliberately conceal their history of HIV infection; thus, medical staff may be exposed to HIV in a relatively long window phase ${ }^{[14,15]}$. Therefore, risks of medical staff to HIV infection and cross infection in hospitals increases immensely when needle sticks or blood splashing occur, and when medical workers fail to receive effective treatment to block the virus within $24 \mathrm{~h}$.

To prevent exposure of medical staff to HIV, hospitals should intensify \occupational safety education and propaganda and deliver relevant trainings to medical staff before they take up their posts. Relevant agencies should establish sound programs to prevent exposure and 
implement them in medical activities. These agencies should also urge relevant administrative departments of hospitals at all levels to intensify health and risk prevention education to medical staff, focus on trainings on professional knowledge, and improve occupational protection and insurance system and monitoring system.

Medical staff must also consciously adopt standard protective measures during medical practice, especially to prevent needle sticks, strictly execute safety operation procedures, and normalize operational acts. These individuals should focus on prevention when they make contact with infectious substances. Medical staff should wash their hands before and after any operation and wear effective filtering medical masks. When handling body fluids or blood, gloves and double-layer gloves must be worn if deemed necessary. During operations, medical workers should strictly abide by requirements. Used pins must not be reused to prevent infection caused by improper operation. Medical wastes should be well classified and disposed of to prevent punctures during disposal. When any medical worker is exposed to HIV during work, he must deal with the wound immediately and consult with professionals to receive blockade treatment. When medical workers are hurt during medical activities by HIV-polluted sharp device, they must debride and sterilize the wound and immediately adopt other protective measures and report the incident to the management department of hospitals in time.

Exposure of medical staff to HIV often occurs incidentally. Thus, their wounds cannot be treated in time. Therefore, hospitals need to intensify drug prophylaxis, apply anti-HIV drugs in time as preventive measure after identifying HIV exposure incidents, and conduct necessary examinations and follow-up visits. Hospitals should also obtain enough information on diseases of outpatients and inpatients and conduct admission examinations in time, especially when obtaining information about HIV and other blood-borne pathogen infections, to gain insights into blood-borne pathogen infection conditions, shorten the window phase for HIV exposure, and reduce HIV exposure risks.

\section{Declarations}

\section{Acknowledgements}

No.

\section{Competing interests}

The authors declare that they have no competing interest.

\section{Authors' contributions}

WL He, C Wen, XY Wang made the literature analysis and wrote, discussed and revised the manuscript of this review. WL He critically analyzed and corrected the manuscript. All authors read and approved the final manuscript.

\section{References}

1 Chen X, Xu C, Chen Q, et al. Investigation Analysis of 156 Medical Workers' Occupational Exposure and Management Countermeasures. Chinese Journal of Nosocomiology, 2011, 21(7): 1390-2.

2 Assessment of Chinese AIDs Epidemic Situation 2011 jointly made by Ministry of Health of PRC, UNAIDS and WHO, Chinese Journal AIDS \& STD $2012,18(1): 1-5$.

3 Chai Y, Zhang J, Song Y, Analysis of Comprehensive Hospitals' Test Results of HIV Antibody and TPPA, Chinese Journal of Human Sexuality, 2013, 22( 1):36-39.

4 Prevention Guideline of Occupational Exposure to Blood-borne Pathogen for Medical Staff of PCR, National Health and Family Planning Commission of PRC. 2009

5 Frijstein G, Hortensius J, Zaaijer H L. Needle stick injuries and infectious patients in a major academic medical centre from 2003 to 2010 . Neth J Med, 2011 69(10):465-8.

6 Li Y, Zhang Z, Wu N, et al. Investigation Analysis of Medical Staff's Occupational Exposure Prevention Status Quo and Countermeasures. Journal of Nurses Training, 2012, 27(6): 504-7.

7 Li W, Zou Y, Zhen Y, et al. Investigation Analysis of Medical Staff' s Occupational Exposure in a Grade 3 Hospital, Chinese Journal of Infection Control, 2015,14(9): 633-5.

8 Xiang $\mathrm{P}, \mathrm{Zhu} \mathrm{H}$, Wang Y, et al. Investigation Analysis of Medical Staff's Occupational Exposure in Comprehensive Hospital and Countermeasures. Chinese Journal of Nosocomiology, 2014, 24(23): 5963-4.

9 Wang J, Yang L. Prevention of Occupational Exposure to Medical Staff. Chinese Journal of Nosocomiology, 2012, 22(4): 763-5.

10 Wang Y, Long Y, Yuan X. Investigation Analysis of Medical Staff's Occupational Exposure Status Quo and Countermeasures. Chinese Journal of Nosocomiology, 2010, 20 (19): 3013-4.

11 Song X, You J, Jin L. Occupational Exposure of Medical Staff to Bloodborne Pathogen and Prevention. China Occupational Medicine, 2016, 4(43):491-4.

12 Lou Y, Lin P, Fu X. Analysis of Occupational Exposure to HIV in Guangdong Province during 2008-2010. Chinese Journal of AIDS \& STD, 2012, 18(10): 653-6. 
13 Wei C. Occupational Exposure of Nurses to HIV and Its Preventions and Treatment. Journal of Public Health and Preventive Medicine, 2016, 17(67):67-9.

14 Liu Y, Zhang L, Guo K, et al. Occupational Exposure to Blood-borne Pathogens in Medical Personnel. Chinese Journal of Nosocomiology,
2011,21(9): 1809-1810.

15 Liu X. Investigation Analysis of Occupational Exposure of 28 Medical Workers to Blood-borne Pathogen. Laboratory Medicine and Clinic, 2010, 7 (2): 159-160. 